\title{
E-cadherin Immunohistochemistry Stain and Acute Erythroid Leukemia, the Emerging Story
}

\section{Mansour SA*}

King Khalid University Hospital - King Saud University, Kingdom of Saudi Arabia

*Corresponding author: Mansour S Aljabry, Hematology unit- Pathology department, King Khalid University Hospital, King Saud University, Kingdom of Saudi Arabia, Tel: +966114671812; E-mail: m.aljabry4210@gmail.com

Rec date: Jul 11, 2015; Acc date: Aug 04, 2015; Pub date: Aug 06, 2015

Copyright: ( 2015 Mansour SA. This is an open-access article distributed under the terms of the Creative Commons Attribution License, which permits unrestricted use, distribution, and reproduction in any medium, provided the original author and source are credited.

\begin{abstract}
E-Cadherin is calcium-dependent cell adhesion molecule encoded by CDH1 gene at chromosome 16q22.1. Ecadherin regulates cell-cell adhesions and controls mobility and proliferation of epithelial cells. Recently, the role of E-cadherin in erythroid maturation has been elucidated. It is selectively expressed in immature erythroblasts and gradually down regulated as erythroid precursors mature. Acute erythroid leukemia is neoplastic proliferation of immature erythroid precursors in bone marrow with or without significant myeloblasts component. In the recent years E-cadherin has emerged as the most sensitive and specific marker for immature erythroblasts. Moreover, combination of E-cadherin with glycophorin A in immunohistochemistry staining can effectively separate immature vs. mature erythroid precursors in bone marrow biopsy. In this short review I will shed light on the key points related to the nature of relationship between E-cadherin and acute erythroid leukemia.
\end{abstract}

Keywords: Bone marrow; Down regulated; E-cadherin; Immunohistochemistry; Precursors

\section{Introduction}

\section{E-cadherin}

E-cadherin (epithelial cadherin) is a polypeptide protein belongs to cadherins family whose primary function is to facilitate cell-cell adhesion and recognition [1]. Cadherins are class-1 transmembrane proteins named for "calcium-dependent adhesion" because they are dependent on calcium $\left(\mathrm{Ca}^{2+}\right)$ ions to function [2].

E-cadherin is encoded by CDH1 gene which is located on the long (q) arm of chromosome 16 at position 22.1. [3] It is mainly expressed on epithelial cells and behaves as both receptor and ligand for other molecules and can be used as a marker for epithelial differentiation owing to limited expression to the cells of epithelial origin. In addition to that, E-cadherin has a major role in the morphogenesis of cells during migration and acts as tumor suppressor gene [4,5]. In some solid tumors, such as breast cancer, loss of E-cadherin function is often associated with tumor progression and transition to more invasive disease [6].

\section{Acute erythroid leukemia (AEL)}

AEL is a unique type of acute myeloid leukemia characterized by proliferation of immature erythroid precursors (typically $>50 \%$ ) [7]. AEL is divided into two subtypes based on presence or absence of a significant myeloid component: Erythroleukemia and pure erythroid leukemia erythroleukemia (erythroid/myeloid) is characterized by marked erythroid proliferation ( $\geq 50 \%$ of the entire BM cells) accompanied with significant number of myeloblasts $(\geq 20 \%$ of nonerythroid cells). Typically, the erythroid component is composed of all stage of maturation with shift to immaturity and significant dysplastic features such as megaloblastoid changes and multi nucleation. Pure erythroid leukemia is neoplastic proliferation of proerythroblasts (the early recognizable erythroid precursors) and other immature erythroid precursors comprising at least $80 \%$ of bone marrow cells. Myeloblasts are not detected in this subtype [7-9].

Erythroblasts express erythrocyte associated antigens; this expression correlates with the degree of differentiation or maturation of the neoplastic cells. Erythroblasts usually express CD71 (transferrin receptor) hemoglobin $\mathrm{A}$, glycophorin $\mathrm{A}, \mathrm{ABH}$ blood group antigens, and HLA-DR. Occasionally, expression of CD71 and glycophorin A can be aberrantly dim or often negative especially in immature stages of erythropoiesis. Erythroid precursors are classically negative for myeloid associated antigens such as CD13, CD33, and MPO. Immature markers such as CD34 and TDT are usually negative as well [10-12].

\section{The emerging story}

In 1995, Sorin A et al. proved that E-cadherin plays a major role in the maturation of the erythroid lineage [13]. This non anticipated finding was confirmed by western blot (WB) analysis and immunofluorescence staining of bone marrow samples. Dual color flow cytometry revealed that the expression of E-cadherin is restricted to immature erythroid precursor (pro-erythroblasts and basophilic erythroblasts) while more mature erythroid precursors are negative. Interestingly, Erythropoiesis was dramatically diminished when antiE-cadherin antibody was added to bone marrow culture .It is postulated that E-cadherin has a major role in establishment of an appropriate microenvironment necessary for erythroid maturation [14].

The subsequent studies highlighted the significance of this finding [15-17]. G Acs et al showed that E-cadherin was strongly expressed in normal erythroid precursors of normal bone marrow specimens. In contrast, erythroblasts in erthroleukemia (erythroid/myeloid leukemia) lack membrane E-cadherin expression. However, the majority of erythroleukemia cases expressed E-cadherin as a Golgi 
distribution pattern, but not as cell surface pattern. Additionally, Ecadherin expression was stronger in more immature erythroid precursors and down regulated during erythroid maturation [18].

In the research of Liu et al., E-cadherin showed weak-to strong membrane reactivity in sheets of large, immature erythroblasts in 13 of 14 cases $(93 \%)$ of pure erythroid leukemia, while Glycophorin A stained mainly the mature erythroblasts but was weakly positive in the immature erythroblasts (21\%). The combination of E-cadherin with glycophorin effectively separated immature vs. mature erythroid precursors in bone marrow biopsy section. Moreover, the variability in the staining pattern (Golgi vs. membranous) is demonstrated in normal and abnormal erythroid precursors [19]. This variability makes a distinction between immature and mature erythroid precursors based on E-cadherin expression alone is a bet difficult.

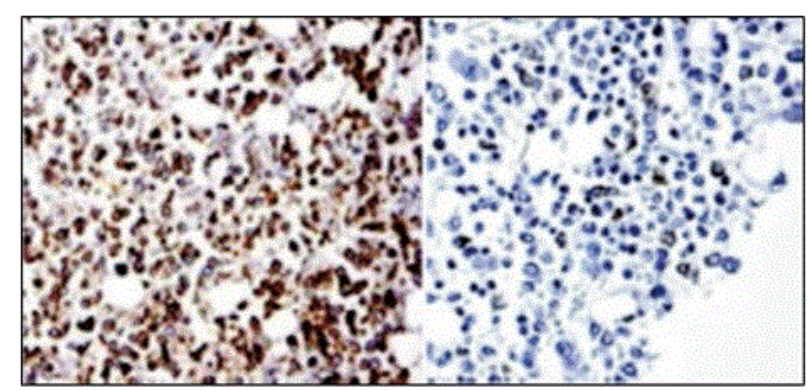

Figure 1: Comparison between E-cadherin and Glycophorin A of bone marrow biopsy in pure erythroid leukemia 20 (H\&E 100X): A: Immature erythroid are strongly positive for E-cadherin. B: Glycophorin A shows weak positivity.

In a unique study, Robert et al. examined 84 cases, including almost all acute myeloid leukemia subtypes, in order to determine the specificity of E-cadherin for leukemic blasts [14]. Of these 84 cases, six showed significant positivity $(>50 \%)$ for E-cadherin; 5 cases of acute erythroid leukemia and one megakaryoblastic leukemia associated with Down syndrome which was positive as well. Of the five cases of acute erythroid leukemia, one case was pure erythroid leukemia (PEL) and the rest were acute erythroid myeloid leukemia cases. The only case which was positive for E-cadherin and did not belong to acute erythroid leukemia expressed other markers of erythroid differentiation such as glycophorin A. This case is likely to have proliferation of both malignant erythroblasts and megakaryoblasts however; no certain conclusion can be obtained.

Our local experience with E-cadherin is in agreement with the published literature. We received 2 cases of pure erythroid leukemia in the last 3 years. The first case was for middle age patient who diagnosed with pure erythroid leukemia after long history of alcohol abuse [20]. Immunohistochemistry for this case showed strong positivity $(\approx 80 \%)$ to E-cadherin (ECH-6; Ventana Medical Systems, Inc., Roche, Basel, Switzerland), weak positivity $(\approx 20 \%)$ for glycophorin A (GA-R2; Ventana Medical Systems, Inc.) and negative for CD34, MPO, vWF, CD3 and CD79a. In fact, the combination of Ecadherin with glycophorin A distinguished between immature and mature erythroid precursors efficiently (Figure1).

The second case was for 21 year old patient who presented with poorly differentiated blasts in peripheral blood and bone marrow. The infiltrating blasts were completely negative for Glycophorin A and all other myeloid and lymphoid markers including CD41 and CD61. CD36 and CD71 were partially positive. Interestingly, E-cadherin was strongly expressed by almost all immature cells $(\approx 95 \%)$ and was quite helpful in pure erythroid leukemia diagnosis. This patient underwent allogeneic stem cell transplantation but unfortunately, he relapsed after 6 months with circulating erythroblasts and moderate bone marrow fibrosis.

E-cadherin was moderately positive $(\approx 40 \%)$ in immature erythroid precursors. E-cadherin stain is a useful marker that more clearly defines immature erythroid cells compared to glycophorin A [17].

\section{Conclusion}

The utility of E-cadherin stain as specific and sensitive marker for erythroid differentiation has been elucidated in reasonable number of cases. Moreover, it is useful marker that distinguishes pure erythroid leukemia from other erythroid proliferation. Molecular studies, preferably using high-throughput methods, are needed to clarify the nature of the relationship between abnormal expression of E-cadherin and tumorigenesis in acute erythroid leukemia.

\section{References}

1. Van RF, Berx G (2008) The cell-cell adhesion molecule E-cadherin. Cell Mol Life Sci 65: 3756-3788.

2. Stockinger A, Eger A, Wolf J, Beug H, Foisner R (2001) E-cadherin regulates cell growth by modulating proliferation-dependent betacatenin transcriptional activity. J Cell Biol154: 1185-1196.

3. John RM, Paul CV, Ross DB, Susan JC (2000) Hyper methylation of Ecadherin in leukemia. Blood 95: 10

4. Pecina SN (2003) Tumor suppressor gene E-cadherin and its role in normal and malignant cells. Cancer Cell Int 3: 17.

5. Rajwar YC, Jain N, Bhatia G, Sikka N, Garg B et al. (2015) Expression and Significance of Cadherins and Its Subtypes in Development and Progression of Oral Cancers: A Review. J Clin Diagn Res 9: ZE05-ZE07.

6. Canel M, Serrels A, Frame MC (2013) E-cadherin-integrin crosstalk in cancer invasion and metastasis. J Cell Sci 126:393-401.

7. Swerdlow SH (2008) International Agency for Research on Cancer, World Health Organization WHO Classification of Tumours of Haematopoietic and Lymphoid Tissues. IARC Press, Lyon, France.

8. Bain BJ (2013) Di Guglielmo and his syndromes. Br J Haematol 120: 939-943.

9. Goldberg SL, Noel P, Klumpp TR, Dewald GW (1998) The erythroid leukemias: a comparative study of erythroleukemia (FAB M6) and Di Guglielmo disease. Am J Clin Oncol 21: 42-47.

10. Kowal VA, Mazzella FM, Cotelingam JD (2000) Diagnosis and characterization of acute erythroleukemia subsets by determining the percentages of myeloblasts and proerythroblasts in 69 cases. Am J Hematol 65: 5-13.

11. Olufunmilayo O, Thangavelu M, Larson RA (1992) Clinical, morphologic and cytogenetic characteristics of 26 patients with acute erythroblastic leukemia. Blood 80: 2873-2882.

12. Brunning RD, Matutes E, Flandrin G (2001) Acute myeloid leukemia not otherwise categorized. In: Jaffe ES, Harris NL, Stein H, Vardiman JW, eds. Pathology and Genetics of Tumours of Haematopoietic and Lymphoid Tissues. IARC Press, Lyon, France, pp. 97-99.

13. Armeanu S, Buhring HJ, Reuss-Borst M, Muller CA, Klein G (1995) Ecadherin is functionally involved in the maturation of the erythroid lineage. J Cell Biol 131:243.

14. Robert SO, Karen M (2014) E-Cadherin is a specific marker for erythroid differentiation and has utility, in combination with CD117 and CD34, for 
Citation: Mansour SA (2015) E-cadherin Immunohistochemistry Stain and Acute Erythroid Leukemia, the Emerging Story. J Cytol Histol 6: 352. doi:10.4172/2157-7099.1000352

Page 3 of 3

enumerating myeloblasts in hematopoietic neoplasms. Am J Clin Pathol 141: 656-664.

15. Armeanu S, MÜller CA, Klein G (2000) Involvement of E-cadherin in the Development of Erythroid Cells, Subject Heading. Hematol 5: 307-316.

16. Corn PG, Heath EI, Heitmiller R, Fogt F, Forastiere AA, et al. (2001) Frequent hyper methylation of the $59 \mathrm{CpG}$ island of E-cadherin in esophageal adenocarcinoma. Clin Cancer Res 7: 2765-2769.

17. Morosan C, Allan RW (2008) E-Cadherin Immunohistochemical Stain Is a Useful Specific Marker of Early Erythroid Elements in Bone Marrow
Core Biopsies and Has Utility in MDS and Erythroleukemia. Mod Pathol 21: 267A.

18. Acs G, LiVolsi VA (2001) Loss of membrane expression of E-cadherin in leukemic erythroblasts. Arch Pathol Lab Med 125: 198-201.

19. Liu W, Hasserjian RP, Hu Y (2011) Pure erythroid leukemia: a reassessment of the entity using the 2008 World Health Organization classification. Mod Pathol 24: 375-383.

20. Mansour Aljabry (2015) Complex karyotype with novel translocation in pure erythroid leukemia patient. Hematol Rep 7: 5674. 